\title{
Research on the Evaluation Model of Urban Tourism Management Efficiency with Uncertain Linguistic Information
}

\author{
Song-Mao Wang, ${ }^{1}$ Liang-Yan Fang, ${ }^{2}$ and Feng Deng ${ }^{1}$ \\ ${ }^{1}$ Xinjiang University, Urumqi, Xinjiang 830047, China \\ ${ }^{2}$ Urumqi Vocational University, Urumqi, Xinjiang 830047, China \\ Correspondence should be addressed to Song-Mao Wang; wsm0316@sohu.com
}

Received 29 June 2014; Accepted 9 July 2014; Published 17 July 2014

Academic Editor: Xiaofei Zhao

Copyright (C) 2014 Song-Mao Wang et al. This is an open access article distributed under the Creative Commons Attribution License, which permits unrestricted use, distribution, and reproduction in any medium, provided the original work is properly cited.

We investigate the multiple attribute decision making problems for evaluating the urban tourism management efficiency with uncertain linguistic information. We utilize the uncertain linguistic weighted averaging (ULWA) operator to aggregate the uncertain linguistic information corresponding to each alternative and get the overall value of the alternatives and, then rank the alternatives and select the most desirable one(s). Finally, a numerical example for evaluating the urban tourism management efficiency with uncertain linguistic information is used to illustrate the proposed model.

\section{Introduction}

China's tourism industry has experienced the stages of formation, rapid growth, and steady development since 1978 and now has developed into one of the fastest growing industries and a new growth point of China's national economy. As the core and foundation of the development of China's tourism industry, urban tourism has made an important contribution to the rapid prosperity and growth of China's tourism industry in the past 35 years $[1,2]$. The industrial status of tourism industry in the urban economy has risen to become a key industry and one of the pillar industries of urban tertiary industry and has also become the core of urban vigorous development of tertiary industry so as to optimize the urban industrial structure $[3,4]$. So in-depth study of the development of urban tourism industry is not only the objective requirement of promoting the development of china's tourism industry but also the reality need of optimizing the allocation of the tourism system so as to optimize the urban industrial structure and promote the urban economic growth. Although the overall urban tourism industry has shown a scene of prosperity and development, we are concerned about the differences in the development of urban tourism industry, especially the regional differences in tourism industry of the eastern, central, and western cities showing the different characteristics of the development $[5,6]$. The essence of these different characteristics is the macroperformance under the influence of microscopic mechanism of the development of the tourism industry. Therefore, we can analyse the microinfluencing factors under the macroscopic characteristics of the development of urban tourism industry in order to study the breakthrough point to grasp, refine, and identify the mode of development of urban tourism of the growth center of eastern, central, and western regions. To make out what factors are the key factors which contribute to the urban development of the tourism industry and what factors are the restrictive factors which hinder the development of the tourism industry in different development modes $[7,8]$. Distinguishing the mode of development of urban tourism with a breakthrough point of its factors and refining and summarizing the characteristics of the development paths and effects of urban tourism industry in the growth centers of eastern, central, and western cities will play a demonstration effect for the other cities with similar industrial development conditions in the eastern, central, and western regions and provide reference of development mode and development path selection for other cities to improve the tourism industry system and enhance and optimize the structure of tourism industry $[9,10]$. 
In this paper, we investigate the multiple attribute decision making problems $[11,12]$ for evaluating the urban tourism management efficiency with uncertain linguistic information. We utilize the uncertain linguistic weighted averaging (ULWA) operator to aggregate the uncertain linguistic information corresponding to each alternative and get the overall value of the alternatives and then rank the alternatives and select the most desirable one(s). Finally, a numerical example for evaluating the urban tourism management efficiency with uncertain linguistic information is used to illustrate the proposed model.

\section{Preliminaries}

Let $S=\left\{s_{i} \mid i=1,2, \ldots, t\right\}$ be a linguistic term set with odd cardinality. Any label, $s_{i}$, represents a possible value for a linguistic variable, and it should satisfy the following characteristics $[13,14]$ : (1) the set is ordered $s_{i}>s_{j}$, if $i>j$; (2) there is the negation operator $\operatorname{neg}\left(s_{i}\right)=s_{j}$ such that $j=t+1-i$. For example, $S$ can be defined as

$$
\begin{aligned}
S=\left\{s_{1}\right. & =\text { extremely poor, } s_{2}=\text { very poor, } \\
s_{3} & =\text { poor, } s_{4}=\text { medium, } s_{5}=\text { good }, \\
s_{6} & \left.=\text { very good, } s_{7}=\text { extremely good }\right\} .
\end{aligned}
$$

Definition 1 (see [15]). Let ULWA : $\widetilde{S}^{n} \rightarrow \widetilde{S}$; if

$$
\operatorname{ULWA}_{\omega}\left(\widetilde{s}_{1}, \widetilde{s}_{2}, \ldots, \widetilde{s}_{n}\right)=\omega_{1} \times \widetilde{s}_{1} \oplus \omega_{2} \times \widetilde{s}_{2} \oplus \cdots \omega_{n} \times \widetilde{s}_{n},
$$

where $\omega=\left(\omega_{1}, \omega_{2}, \ldots, \omega_{n}\right)$ is the weighting vector of uncertain linguistic variables $\widetilde{s}_{i}\left(\widetilde{s}_{i} \in \widetilde{S}, i=1,2, \ldots, n\right)$ with $\omega_{i} \in[0,1], \sum_{i=1}^{n} \omega_{i}=1$, then the function ULWA is called the uncertain linguistic weighted averaging (ULWA) operator of dimension $n$.

Definition 2 (see [15]). Let $\widetilde{s}_{1}=\left[s_{\alpha_{1}}, s_{\beta_{1}}\right]$ and $\widetilde{s}_{2}=\left[s_{\alpha_{2}}, s_{\beta_{2}}\right]$ be two uncertain linguistic variables, and let len $\left(\widetilde{s}_{1}\right)=\beta_{1}-\alpha_{1}$ and len $\left(\widetilde{s}_{2}\right)=\beta_{2}-\alpha_{2}$, and then the degree of possibility of $\widetilde{s}_{1} \geq \widetilde{s}_{2}$ is defined as [14]

$$
p\left(\widetilde{s}_{1} \geq \widetilde{s}_{2}\right)=\frac{\max \left(0, \operatorname{len}\left(\widetilde{s}_{1}\right)+\operatorname{len}\left(\widetilde{s}_{2}\right)-\max \left(\beta_{2}-\alpha_{1}, 0\right)\right)}{\operatorname{len}\left(\widetilde{s}_{1}\right)+\operatorname{len}\left(\widetilde{s}_{2}\right)} .
$$

\section{Research on the Evaluation Model of Urban Tourism Management Efficiency with Uncertain Linguistic Information}

In this section, we investigate the multiple attribute decision making problems for evaluating the urban tourism management efficiency with uncertain linguistic information. Let $A=\left\{A_{1}, A_{2}, \ldots, A_{m}\right\}$ be a discrete set of alternatives and $G=\left\{G_{1}, G_{2}, \ldots, G_{n}\right\}$ the set of attributes; $\omega=\left(\omega_{1}, \omega_{2}, \ldots, \omega_{n}\right)$ is the exponential weighting vector of the attributes $G_{j}(j=$ $1,2, \ldots, n)$, where $\omega_{j} \in[0,1], \sum_{j=1}^{n} \omega_{j}=1$. Suppose that $\widetilde{R}=\left(\widetilde{r}_{i j}\right)$ is the decision matrix, where $\widetilde{r}_{i j} \in \widetilde{S}$ are the uncertain linguistic variables.
In the following, we apply the ULWA operator to MADM forevaluating the urban tourism management efficiency with uncertain linguistic information.

Step 1. Utilize the decision information given in matrix $\widetilde{R}$ and the ULWA operator

$$
\begin{array}{r}
\widetilde{r}_{i}=\operatorname{ULWA}_{\omega}\left(\widetilde{r}_{i 1}, \widetilde{r}_{i 2}, \ldots, \widetilde{r}_{i n}\right), \\
i=1,2, \ldots, m,
\end{array}
$$

to derive the collective overall preference values $\widetilde{r}_{i}(i=$ $1,2, \ldots, m)$ of the alternative $A_{i}$, where $\omega=\left(\omega_{1}, \omega_{2}, \ldots, \omega_{n}\right)^{T}$ is the weighting vector of the attributes.

Step 2. To rank these collective overall preference values $\widetilde{r}_{i}$ $(i=1,2, \ldots, m)$, we first compare each $\tilde{r}_{i}$ with all the $\tilde{r}_{j}$ $(j=1,2, \ldots, m)$ by using (2). For simplicity, we let $p_{i j}=$ $p\left(\widetilde{r}_{i} \geq \widetilde{r}_{j}\right)$, and then we develop a complementary matrix as $P=\left(p_{i j}\right)_{m \times m}$, where

$$
\begin{gathered}
p_{i j} \geq 0, \quad p_{i j}+p_{j i}=1, \\
h p_{i i}=0.5, \quad i, j=1,2, \ldots, m .
\end{gathered}
$$

Summing all the elements in each line of matrix $P$, we have

$$
p_{i}=\sum_{j=1}^{m} p_{i j}, \quad i=1,2, \ldots, m
$$

Step 3. Rank all the alternatives $A_{i}(i=1,2, \ldots, m)$ and select the best one(s) in accordance with $p_{i}(i=1,2, \ldots, m)$.

Step 4. End.

\section{Illustrative Example}

With the rapid development of world tourism, the phenomena of cities "tourlized" and urbanization of tour destination are becoming more and more prominent. All these promote the function and facilities of cities to cater for a urban tourist in the course of urban plan and construction, which meet the requirement of tourism and meanwhile form the convergence and development of tourist economic elements. As the carrier of tourist development, tourist urban management is the key problem to be solved in the urban development of our country, and it would bring significant influence to the development of economy and society of our country. In this section, we present an empirical case study of evaluating the urban tourism management efficiency with uncertain linguistic information. There is a panel with five possible urban tourism cities $A_{i}(i=1,2,3,4,5)$ to select. The team of experts must take a decision according to the following four attributes: (1) $G_{1}$ is the informatization level; (2) $G_{2}$ is the financial level; (3) $G_{3}$ is the service level; (4) $G_{4}$ is the business level. The five possible urban tourism cities $A_{i}(i=$ $1,2,3,4,5)$ are to be evaluated using the linguistic term set $S$ by the decision makers under the above four attributes (whose weighting vector $\omega=(0.20,0.30,0.40,0.10)^{T}$ ) and 
construct, respectively, the decision matrix as follows: $\widetilde{R}=$ $\left(\widetilde{r}_{i j}\right)_{5 \times 4}$ and

$$
\left.\begin{array}{ccccc} 
& G_{1} & G_{2} & G_{3} & G_{4} \\
A_{1} & \left(\left[s_{4}, s_{6}\right]\right. & {\left[s_{5}, s_{6}\right]} & {\left[s_{3}, s_{4}\right]} & {\left[s_{2}, s_{4}\right]} \\
A_{2} & {\left[s_{2}, s_{4}\right]} & {\left[s_{2}, s_{3}\right]} & {\left[s_{4}, s_{5}\right]} & {\left[s_{4}, s_{5}\right]} \\
A_{3} & {\left[s_{4}, s_{5}\right]} & {\left[s_{3}, s_{5}\right]} & {\left[s_{1}, s_{2}\right]} & {\left[s_{5}, s_{6}\right]} \\
A_{4} & {\left[s_{5}, s_{6}\right]} & {\left[s_{4}, s_{5}\right]} & {\left[s_{4}, s_{6}\right]} & {\left[s_{2}, s_{3}\right]} \\
A_{5} & {\left[s_{3}, s_{4}\right]} & {\left[s_{5}, s_{6}\right]} & {\left[s_{3}, s_{5}\right]} & {\left[s_{5}, s_{6}\right]}
\end{array}\right)
$$

In the following, we apply the ULWA operator to MADM forevaluating the urban tourism management efficiency with uncertain linguistic information. To get the most desirable urban tourism cities, the following steps are involved.

Step 1. Utilizing the ULWA operator, we obtain the collective overall preference values $\widetilde{r}_{i}$ of the urban tourism cities $A_{i}(i=$ $1,2,3,4,5)$

$$
\begin{array}{lll}
\tilde{r}_{1}=\left[s_{3.21}, s_{5.18}\right], & \widetilde{r}_{2}=\left[s_{3.74}, s_{4.82}\right], & \widetilde{r}_{3}=\left[s_{4.72}, s_{5.46}\right], \\
\tilde{r}_{4}=\left[s_{3.36}, s_{5.57}\right], & \tilde{r}_{5}=\left[s_{4.62}, s_{6.03}\right] . &
\end{array}
$$

Step 2. Rank these overall preference values $\widetilde{r}_{i}(i=1,2,3,4,5)$; we first compare each $\widetilde{r}_{i}$ with all the $\widetilde{r}_{j}(j=1,2,3,4,5)$ by using (3) and then develop a complementary matrix:

$$
P=\left[\begin{array}{lllll}
0.500 & 0.720 & 0.180 & 0.459 & 0.132 \\
0.280 & 0.500 & 0.010 & 0.350 & 0.390 \\
0.820 & 0.990 & 0.500 & 0.758 & 0.450 \\
0.541 & 0.650 & 0.242 & 0.500 & 0.222 \\
0.868 & 0.610 & 0.550 & 0.778 & 0.500
\end{array}\right] .
$$

Summing all the elements in each line of matrix $P$, we have

$$
\begin{array}{ll}
p_{1}=2.037, & p_{2}=1.108, \quad p_{3}=3.564, \\
p_{4}=2.246, & p_{5}=3.663 .
\end{array}
$$

Step 3. Rank all the urban tourism cities $A_{i}(i=1,2,3,4,5)$ in accordance with the values $p_{i}(i=1,2,3,4,5): A_{5}>A_{3}>$ $A_{4}>A_{1}>A_{2}$. Thus, the most desirable urban tourism city is $A_{5}$.

\section{Conclusions}

With the rapid development of tourism and city, the development of tourism in promoting the city has also brought a series of problems: the integration of tourism resources among the cities is more difficult, lacking of urban tourism regional planning, the chaos of urban tourism functions and other features, tourism functional area is difficult to develop and so on, and the internal region has also suffered more severe competitive pressures. How to cultivate and improve the tourist city of travel services functional areas, in order to improve tourism services, and better development of urban tourism are becoming the most urgent problems to be solved in each tourist city currently. In this paper, we investigate the multiple attribute decision making problems for evaluating the urban tourism management efficiency with uncertain linguistic information. We utilize the uncertain linguistic weighted averaging (ULWA) operator to aggregate the uncertain linguistic information corresponding to each alternative and get the overall value of the alternatives and then rank the alternatives and select the most desirable one(s). Finally, a numerical example for evaluating the urban tourism management efficiency with uncertain linguistic information is used to illustrate the proposed model.

\section{Conflict of Interests}

The authors declare that there is no conflict of interests regarding the publication of this paper.

\section{Acknowledgment}

The work was supported by the Xinjiang Social Science Fund (13BGL035).

\section{References}

[1] X. Wang, "Model for tourism management with 2-tuple linguistic information," Advances in Information Sciences and Service Sciences, vol. 3, no. 4, pp. 34-39, 2011.

[2] C. Yan, "An improved city tourism efficiency evaluation model based on data envelopment analysis," Journal of Digital Content Technology and Its Applications, vol. 7, no. 2, pp. 343-349, 2013.

[3] H. U. Xiaotao, "The application of GIS technology in tourism resources management," JDCTA, vol. 7, no. 3, pp. 308-314, 2013.

[4] G. Xiong and M. Jiang, "The analysis and evaluation on tourism competitiveness of Poyang lake ecological economic zone of china based on TOPSIS method," International Journal of Advanced Computer Technology, vol. 5, no. 4, pp. 720-726, 2013.

[5] W. Min, "Supply chains for tourism enterprises management based on information management system," International Journal of Advancements in Computing Technology, vol. 5, no. 5, pp. 1000-1010, 2013.

[6] G. D. Zhuang and H. Zhang, "Innovation strategies and technology for tourism industry based on case-of-tourism theory," AISS, vol. 5, no. 5, pp. 97-105, 2013.

[7] Y. C. Dong, W. C. Hong, Y. F. Xu, and S. Yu, "Selecting the individual numerical scale and prioritization method in the analytic hierarchy process: a 2-Tuple fuzzy linguistic approach," IEEE Transactions on Fuzzy Systems, vol. 19, no. 1, pp. 13-25, 2011.

[8] Z.-P. Fan, B. Feng, and W.-L. Suo, "A fuzzy linguistic method for evaluating collaboration satisfaction of NPD team using mutual-evaluation information," International Journal of Production Economics, vol. 122, no. 2, pp. 547-557, 2009.

[9] Z. P. Fan and Y. Liu, "A method for group decision-making based on multi-granularity uncertain linguistic information," Expert Systems with Applications, vol. 37, no. 5, pp. 4000-4008, 2010.

[10] Y. Dong, Y. Xu, and H. Li, "On consistency measures of linguistic preference relations," European Journal of Operational Research, vol. 189, no. 2, pp. 430-444, 2008. 
[11] Y. C. Dong, Y. F. Xu, H. Y. Li, and B. Feng, “The OWA-based consensus operator under linguistic representation models using position indexes," European Journal of Operational Research, vol. 203, no. 2, pp. 455-463, 2010.

[12] Z. P. Fan, B. Feng, Y. H. Sun, and W. Ou, "Evaluating knowledge management capability of organizations: a fuzzy linguistic method," Expert Systems with Applications, vol. 36, no. 2, pp. 3346-3354, 2009.

[13] F. Herrera and L. Martínez, "A model based on linguistic 2tuples for dealing with multigranular hierarchical linguistic contexts in multi-expert decision-making," IEEE Transactions on Systems, Man, and Cybernetics B: Cybernetics, vol. 31, no. 2, pp. 227-234, 2001.

[14] F. Herrera and L. Martínez, "A 2-tuple fuzzy linguistic representation model for computing with words," IEEE Transactions on Fuzzy Systems, vol. 8, no. 6, pp. 746-752, 2000.

[15] Z. Xu, "Uncertain linguistic aggregation operators based approach to multiple attribute group decision making under uncertain linguistic environment," Information Sciences, vol. 168, no. 1-4, pp. 171-184, 2004. 

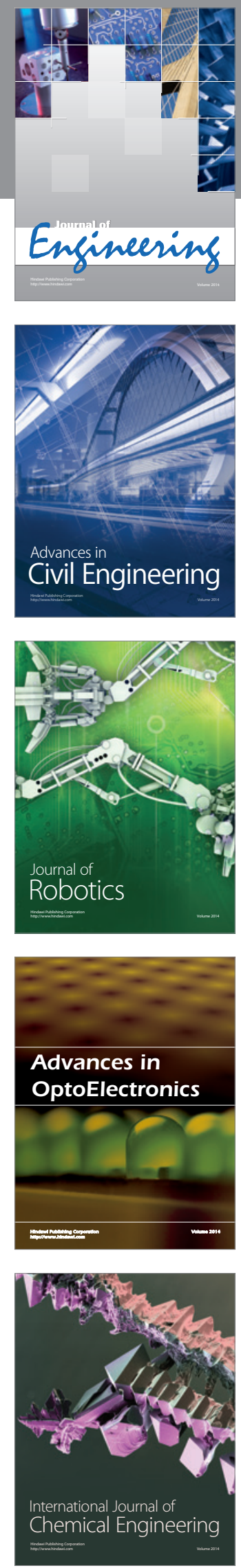

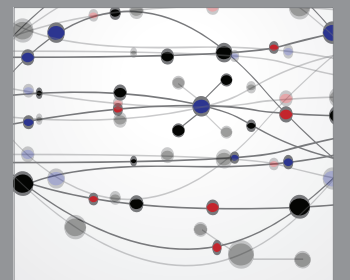

The Scientific World Journal
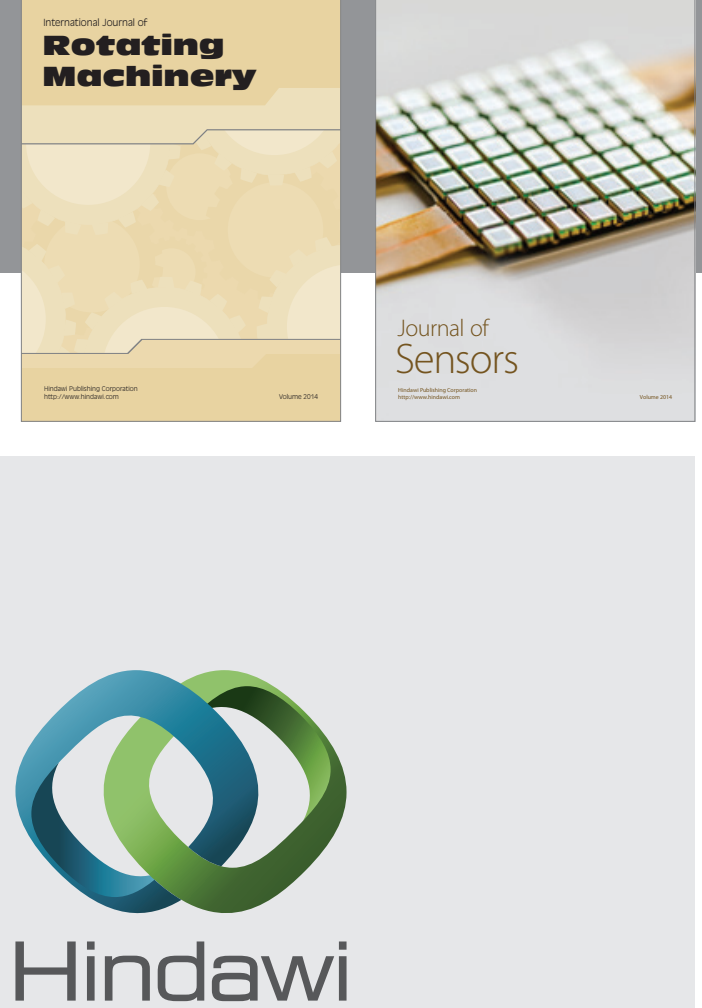

Submit your manuscripts at http://www.hindawi.com
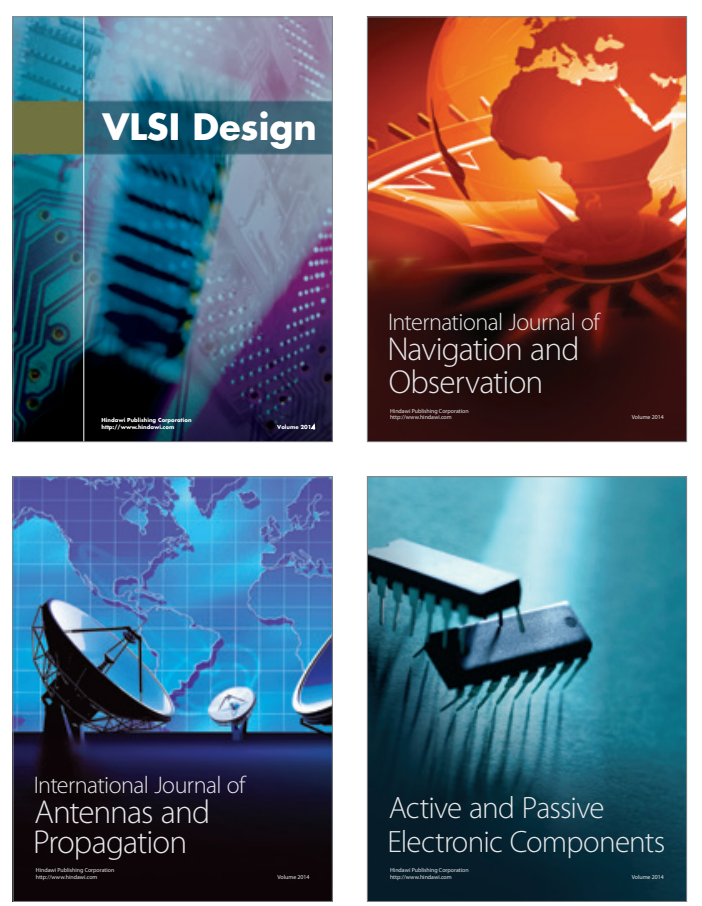
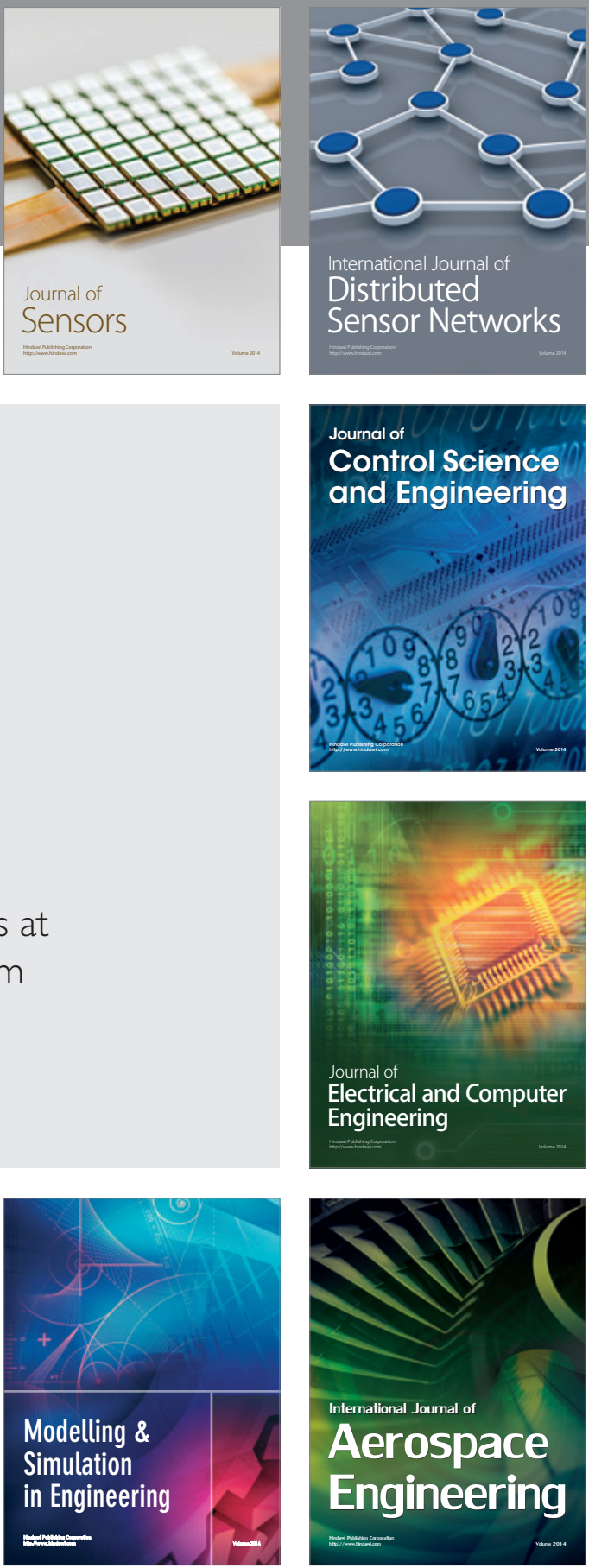

Journal of

Control Science

and Engineering
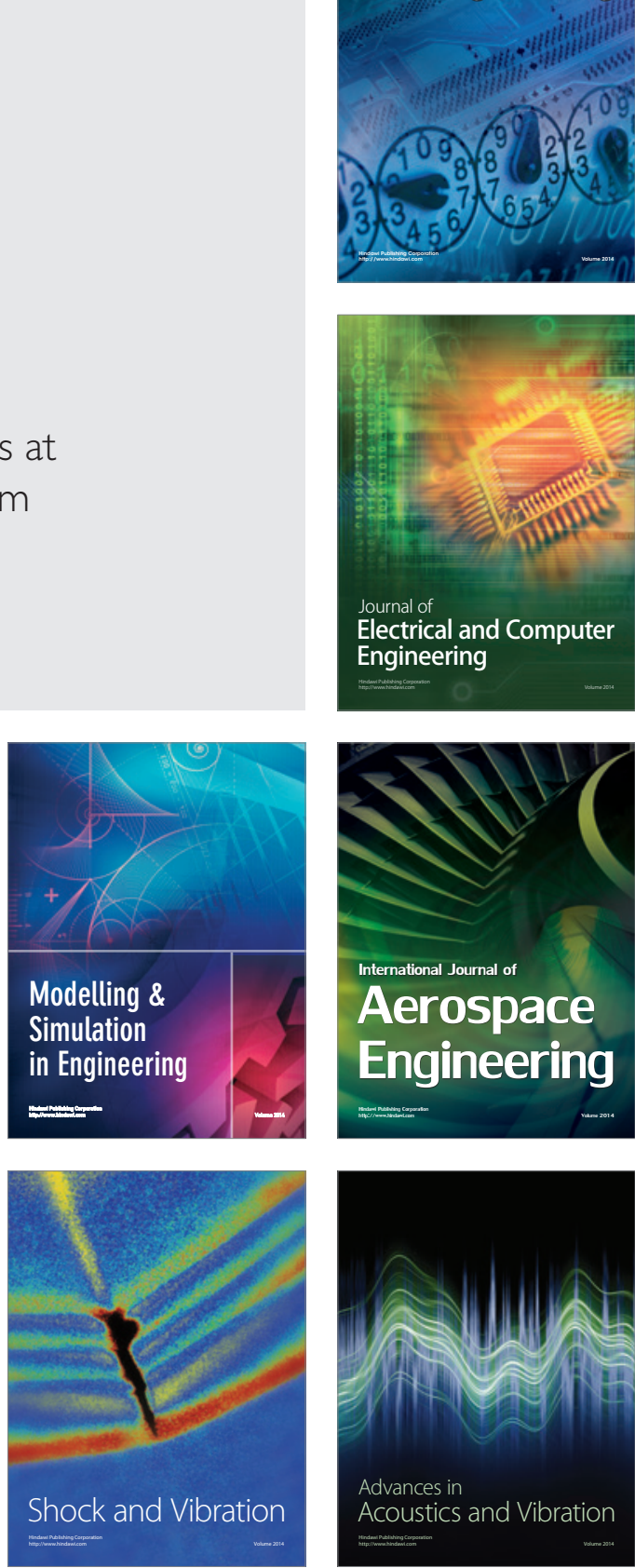